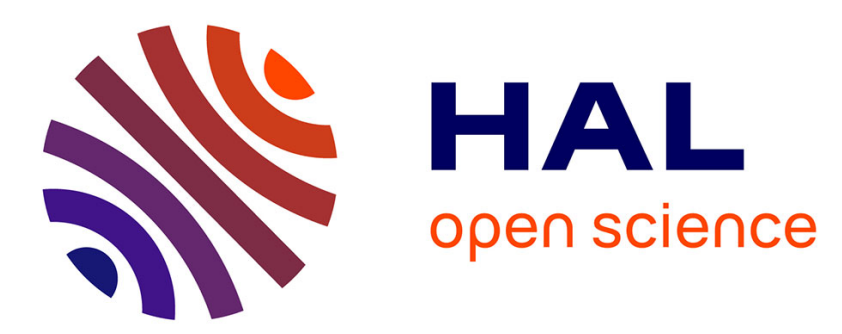

\title{
Analysis of a S-TDMA distributed scheduler for ad-hoc cellular-V2X communication
}

\author{
Laurent Gallo, Jérôme Härri
}

\section{To cite this version:}

Laurent Gallo, Jérôme Härri. Analysis of a S-TDMA distributed scheduler for ad-hoc cellular-V2X communication. Ad Hoc Networks, 2019, 88, pp.160 - 171. 10.1016/j.adhoc.2019.02.002 . hal03486405

\section{HAL Id: hal-03486405 \\ https://hal.science/hal-03486405}

Submitted on 20 Dec 2021

HAL is a multi-disciplinary open access archive for the deposit and dissemination of scientific research documents, whether they are published or not. The documents may come from teaching and research institutions in France or abroad, or from public or private research centers.
L'archive ouverte pluridisciplinaire HAL, est destinée au dépôt et à la diffusion de documents scientifiques de niveau recherche, publiés ou non, émanant des établissements d'enseignement et de recherche français ou étrangers, des laboratoires publics ou privés.

\section{다)(1) $(5$}

Distributed under a Creative Commons Attribution - NonCommerciall 4.0 International 


\title{
Analysis of a S-TDMA Distributed Scheduler for Ad-Hoc Cellular-V2X Communication
}

\author{
Laurent Gallo, Jérôme Härri \\ Communication Systems Dept., EURECOM - 450 Route Des Chappes, 06904 Sophia-Antipolis, France
}

\begin{abstract}
The cellular industry recently proposed a Long Term Evolution (LTE) extension supporting direct Vehicle to Everything (V2X) communication in full ad-hoc mode. Without infrastructure support, a distributed scheduler will play a critical role to the performance of such Cellular-V2X (C-V2X) technology. Self-Organizing TDMA (S-TDMA) is a reliable semi-deterministic and context aware distributed channel access algorithm, which has been considered by European Telecommunications Standard Institute (ETSI) as an attractive alternative to its WiFi-V2X standard, ITS-G5.

In this paper, we describe S-TDMA, and present an analytical model to determine its packet reception performance. We then describe and analyze the performance of a distributed Cellular-V2X scheduler based on S-TDMA. We notably illustrate halfduplex issues impacting its performance in a C-V2X context, and propose two extensions to mitigate them. We finally compare their performance against each other, confirming S-TDMA's potential as distributed scheduler for the future cellular-based V2X technology.
\end{abstract}

Keywords: Vehicle-to-vehicle/roadside/Internet communication, Intelligent Transportation Systems, Network Layer Issues, Unsupervised LTE V2X, Vehicular Safety, Ad-hoc Broadcast

\section{Introduction}

Vehicle to Everything communications will be a fundamental enabler for future autonomous vehicles, which are getting everyday closer to being a reality. V2X communications, in fact, add the cooperative dimension to Cooperative Intelligent Transportation System (C-ITS), enabling wide scale traffic optimization and extending the vehicles' perception beyond the range of their on board sensors. Vehicular communications have hence been a very active subject of research for well over a decade, resulting on the definition of 802.11-based technologies, namely Dedicated Short Range Communications (DSRC) in the US and ITS-G5 in Europe. In recent years, however, a strong push from the automotive and telecommunications industries is promoting cellular-based technologies as candidates to support V2X, under the name of C-V2X.

Automotive applications include a wide set of use cases [1], ranging from collision avoidance, to high density platooning, to highly autonomous driving, each having different requirements in terms of connectivity types and traffic patterns. In particular, V2X technologies will need to support direct Vehicle to Vehicle (V2V) communications, Vehicle to Infrastructure (V2I) and Vehicle to Network (V2N) communications alike. Cellular systems are historically capable of providing connectivity to the network via an interface, referred to as "Uu" interface, linking a User Equipment (UE) to a base station. In the last few years however, the emergence of new use cases and connectivity patterns lead to the definition of Proximity Services (ProSe)

Email addresses: gallo@eurecom.fr (Laurent Gallo), haerri@eurecom.fr (Jérôme Härri) and even more recently LTE-V2X, which enable the support of cellular-based direct device-to-device communications. A new direct UE-to-UE link is defined, referred to as Sidelink (SL), supported by a new "PC5" interface, which directly interconnects two or more terminals.

In this paper, we address direct safety-critical V2V broadcast communications over the PC5 link. This communication paradigm is required for the periodic transmission of status reporting messages, such as Cooperative Awereness Messages (CAMs) in Europe and Basic Safety Messages (BSMs) in the US, which is a fundamental safety feature that must be supported by any V2X technology. By transmitting and receiving these messages, in fact, road users spread and acquire awareness about the instantaneous state of the traffic in their surroundings: it is thus fundamental for these messages to reach as many other road users in proximity as possible, with the shortest possible delay. For this reason, from a spectrum standpoint, the sidelink for $\mathrm{V} 2 \mathrm{~V}$ communications is expected to be deployed on the dedicated 5.9 GHz Intelligent Transportation System (ITS) band, which can be shared for safety applications by every road user, regardless of the operator to which they may be attached. Low latency direct communications between terminals subscribed to different operators, on the other hand, prove challenging from the Radio Resource Management (RRM) perspective. The 3rd Generation Partnership Project (3GPP) specifications define two resource allocation modes, namely "mode 3" and "mode 4". In mode 3, called "network assisted resource allocation", transmissions on the SL are authorized by the network, which provides optimal scheduling. In mode 4 , called "autonomous resource selection", UEs select SL resources au- 
tonomously, which may lead to suboptimal allocations due to collisions. Despite efficiently utilizing channel resources, the mode 3 strictly depends on the network, thus making it difficult for UEs attached to different operators to communicate. This is an issue particularly relevant for the latency-sensitive use case considered in this paper. Hence, the mode 4 is the most convenient choice for this purpose: in this case, the RRM protocol needs to manage the usage of channel resources in a distributed manner, given the absence of a centralized coordination entity.

We propose in this paper to adapt and analyze S-TDMA as distributed RRM for mode 4 LTE V2V. We believe that STDMA is a valid access protocol for mode 4 for several reasons. First, its synchronous slotted channel structure well adapts to the LTE air interface. Second, it has already been extensively studied by means of simulation as an alternate access protocol for $802.11 \mathrm{p}$. It showed equal or better packet reception performance than Carrier Sense Multiple Access with Collision Avoidance (CSMA/CA), while providing stable packet reception performance and predictable channel access delay under any channel load. Finally, it is distributed, and only requires a minimal overhead to be added to each packet.

The contributions of this paper are threefold: we first propose an analytic model to compute the packet-level reception performance of S-TDMA, and show how it inherently provides stability under any load condition. The analytic model enables us to obtain fundamental results which are not dependent on a specific simulation scenario, unlike previous studies. We then evaluate the performance of S-TDMA when it is applied to the LTE V2X channel structure, which, differently from 802.11, enables both time and frequency multiplexing. On the one hand, this time/frequency structure allows cellular-based system to allocate more transmission slots, hence support higher terminal densities. On the other hand however, it poses new challenges due to Half Duplex (HD). We also evaluate S-TDMA over LTE sidelink by means of simulation, highlighting the sources of packet reception error in this configuration. We finally propose and evaluate two novel algorithms, based on modifications of the S-TDMA transmission slot selection mechanism, and which are specifically designed to reduce the impairing effect on reception performance due to half duplex transceivers.

The remainder of this paper is organized as follows: in Section 2 we provide a state of the art of distributed RRM protocols for vehicular communications, after which in Section 3 we illustrate the S-TDMA protocol. The analytical model of STDMA is described in Section 4, and numerically evaluated in Section 5. In Section 6, we discuss the application of S-TDMA to the sidelink air interface, and evaluate its impact on the reception performance; We then propose and evaluate two extensions to STDMA in Section 7. Finally, Section 8 concludes this paper.

\section{Related Work}

Over the years, a wide variety of channel access protocols have been designed to support V2V communications, of which the authors in [2] provide a comprehensive survey. Due to their nature, decentralized algorithms are of particular interest for vehicular networks, and include algorithms such as Vehicular Self Organizing Ad Hoc Networks MAC (VeSOMAC) [3], Vehicular Ad Hoc Networks MAC (VeMAC) [4], and Collision Free Reservation MAC (CFR-MAC) [5]. But in particular, Mobile Slotted Aloha (MS-ALOHA) [6] and S-TDMA [7] are exceptionally relevant protocols, because they both are time slotted, which well fit to the LTE air interface, and have been considered the ETSI as potential alternatives to CSMA/CA [8, 9].

MS-ALOHA [10] is based on two previous Medium Access Control (MAC) schemes, Reliable Reservation ALOHA (RRALOHA) and RR-ALOHA+: MS-ALOHA makes them scalable and more suitable to the vehicular environment. In MSALOHA, an array of fields is piggybacked to each packet, describing how each slot in the current frame is perceived by the transmitter. Furthermore, MS-ALOHA implements a mechanism to control the propagation of such channel usage information, preventing busy slot flags to spread more than two hops. In this way, the hidden terminal problem is averted and the channel utilization is optimized. In [11], the authors of MS-ALOHA compare it by means of simulations against the CSMA/CA mechanism 802.11p is based upon, and against RR-ALOHA+, both in urban and in highway scenarios. It is shown that RR-ALOHA+ poorly adapts to situations, where more nodes are present than channel slots available, which results in remarkable dips in packet reception probability. On the other hand, MS-ALOHA significantly reduces the occurrence of collisions, by providing an efficient slot allocation, which results in better packet reception statistics at ranges within $100 \mathrm{~m}$ when compared to both RRALOHA+ and 802.11p. 802.11p manages to provide shorter channel access delays than MS-ALOHA. However, the latter protocol is able to guarantee a bounded access latency under varying channel loads, whereas this is not the case $802.11 \mathrm{p}$. A qualitative comparison between MS-ALOHA and S-TDMA can be furthermore be found in [12].

3GPP itself recently introduced a listen-before-talk Semi Persistent Scheduling (SPS) distributed algorithm for mode 4 V2V communications, which is visually represented in Fig. 1. In LTE-V2V mode 4, the ITS channel bandwidth is divided into

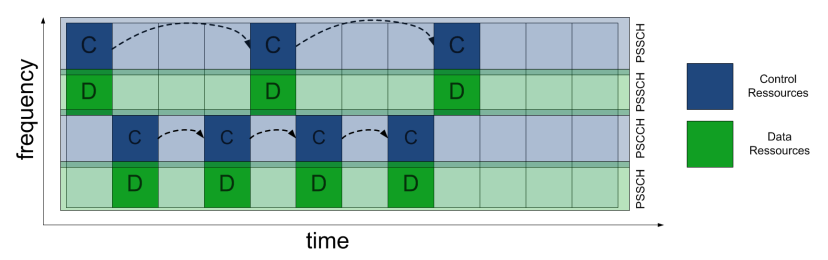

Figure 1: SL Resource Pool for ad hoc resource selection

sub-channels, composed by pairs of control resources and data resources: while the former ones carry the scheduling information, the latter ones transport the corresponding message. In order to minimize latency, the duration of each transmission is one Transmission Time Interval (TTI), equal to one millisecond in LTE. Vehicular UEs constantly sense the occupancy of the channel (over a 100ms window), and schedule their next transmission utilizing resources that are not utilized by other 
users in proximity. The offset to the next transmission is included into the control information of the current transmission, as illustrated in Fig. 1.

S-TDMA operates similarly to SPS, but offers a more flexible structure that improves stability by reserving each transmission slot for multiple subsequent frames. S-TDMA is already commercially adopted for periodical position reporting notably in the shipping industry under the name Automatic Identification System (AIS) [13], and for airborne applications. Given its effectiveness proven on the field, and thanks to extensive research work, such as [7] and [14], the ETSI considered STDMA as a valid alternative to CSMA/CA [8] also for the Automotive industry. Numerous research studies recently focused on S-TDMA. In [15], it is shown to offer a better Packet Reception Probability (PRR) than 802.11p, regardless of the presence of hidden terminals. In [16], the same authors also highlighted its enhanced scalability.

S-TDMA achieves deterministic channel access delay, a significant beneficial feature for safety-related V2X applications, considering that the channel access delay with $802.11 \mathrm{p}$ is known to grow unbounded in high channel load scenarios. For instance, the authors of [14] studied stabilization time of S-TDMA, i.e. the time necessary for the protocol to reach a stable performance. They showed that $802.11 \mathrm{p}$ achieves a low stabilization time (better than S-TDMA) in lightly loaded scenarios, unfortunately increasing as the channel load grows. In this study, STDMA however showed to offer a stable performance independently to the density in the network. Gaugel et al. [17] provided an in-depth evaluation study of the structure of S-TDMA, showing that it offers better performance than 802.11p's CSMA/CA only in ideal scenarios. In [18], however, the same authors showed that the superiority of any of the two protocols over the other is dependent on the considered scenario. This is one of the reasons justifying the need for an analytical model proposed in the first part of this paper, which neither depends on implementations nor on simulation scenarios.

\section{Self-Organizing TDMA}

\subsection{Protocol description}

S-TDMA is a multiple access protocol designed to support periodical transmissions of fixed size messages: for this purpose channel resources are organized into slots with time duration and bandwidth adequate to host a fixed size packet. In this work, we consider CAMs [19], which are periodically transmitted by vehicles to report their current state. Each terminal needs to transmit $r$ packets per second, where $r$ stands for "report rate". In S-TDMA, slots are organized into periodical structures, named "frame", with one second duration, as illustrated in Fig. 2. We denote with $N$ the number of slots that can be hosted within a frame: the value of $N$ depends on the packet size and on transmission parameters such as the modulation and coding scheme, and the bandwidth, which determine the available capacity. It is assumed that terminals, which from now on we will refer to as UEs to conform with LTE nomenclature, are slot synchronous, meaning that the starting and ending instants of each slot must be aligned for all UEs. Frame

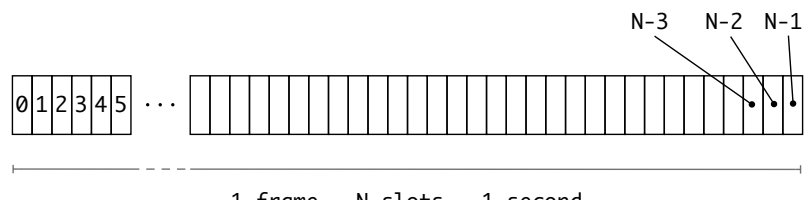

1 frame $=\mathrm{N}$ slots $=1$ second

Figure 2: S-TDMA: channel organization into slots and frames

alignment, on the other hand, is not required. Synchronization can be achieved thanks to Global Navigation Satellite System (GNSS) and maintained by the internal clock as studied in [9]. Furthermore, C-V2X system can also exploit the cellular network synchronization signals to this purpose; synchronization techniques, however, are outside the scope of this work.

The protocol is composed of four phases, which must be performed by each UE after being started up:

1. the initialization phase, during which the UE senses the state of the channel for exactly one frame duration, and builds an internal map representing which of the slots are already being allocated by other UEs;

2. the network entry phase, a short phase (less than one frame) during which the UE performs a one-time transmission of a special Network Entry Packet (NEP), to inform other UEs that it is joining the network;

3. the first frame phase, during which the UE performs the first reservation of its $r$ transmission slots in the first frame;

4. the continuous operations phase, representing the steady state of the protocol, during which the UE transmits CAMs in the previously reserved slots. Furthermore, in order to mitigate the effect of merging collisions, the position of the transmission slots is periodically changed.

The phases will be shortly described in the remainder of this section. For the interested reader, a more detailed description of the protocol can be found in [20].

\subsubsection{The initialization phase}

Right after being started up, a UE enters the initialization phase, during which it listens to the channel for one entire frame. Since no frame synchronization is required, the starting point is randomly dictated by the startup instant. In this process, the state of each slot is registered into an internal map. The possible states are [13]:

- Free: the current slot is not used by any other UE within range;

- Externally Allocated: the current slot is used or reserved for transmission by another UE within RX range;

- Internally Allocated: the current slot is used or reserved for transmission by the current UE. 
- Unavailable: a power level is detected higher than a given Clear Channel Assessment (CCA) threshold, but no information could be correctly decoded. This situation typically happens when multiple packets from UEs within range of the current UE collide, and the Signal to Interference and Noise Ratio (SINR) of either is too low to decode it. This state, proposed in [17], is necessary for implementation purpose.

Internally allocated slots will not be encountered in the initialization phase, since none of them has been reserved yet.

In Fig. 3 a sample scenario is illustrated, wherein the free slots are in white, and the slots detected to be externally allocated are shaded in light blue. The initialization phase terminates when all of the $N$ slots have been listened to, and a state has been assigned to each of them. The UE then switches to the following phase, the network entry phase

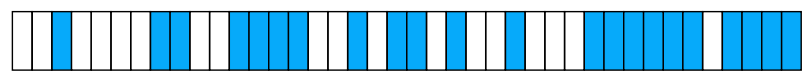

1 frame $=\mathrm{N}$ slots $=1$ second

Figure 3: S-TDMA: initialization phase. Free slots are colored in white, whereas externally allocated slots are shaded in light blue.

\subsubsection{The network entry phase}

The slot for the transmission of the Network Entry Packet is chosen among the free ones using a p-persistent mechanism.

Before transmitting the NEP, the terminal needs to first select the location of the first of its Nominal Transmission Slots (NTSs), i.e. the slot wherein the first CAM packet will be transmitted. In this way, the time offset that separates it from the NEP can be included into the NEP itself, informing other UEs in the network both of the presence of a new terminal and of the position of its first transmission slot.

The procedure to select the first NTS (the number 0), is:

1. a Nominal Interval (NI) is computed, representing the ideal interval between two consecutive transmitted packets. It is defined as $N I=\lfloor N / r\rfloor$, where $r$ is the Report Rate, i.e. the number of transmissions that a terminal needs to perform per second;

2. a Nominal starting Slot (NSS) is randomly chosen among the free slots within the NI following ones. We denote this slot as $\sigma_{n s s}$;

3. a Selection Interval (SI) is defined as the set of slots around the NSS, whose cardinality is determined by the parameter $s$, representing the ratio between the width of the SI and the one of the NI, with $0<s \leq 1$. $s$ is a system parameter, which in AIS is fixed to $s=0.2$. Denoting with $\sigma_{0}, \ldots, \sigma_{N-1}$ the slots within each frame as numbered by the current terminal, the set of the slots belonging to the first SI $\left(S I_{0}\right)$ is given by (1).

$$
\begin{array}{r}
\left.S I_{0}=\left\{\sigma_{(j} \bmod N\right)\right\}, \quad \text { where: } \\
\sigma_{n s s}-\lfloor N s /(2 r)\rfloor \leq j \leq \sigma_{n s s}+\lfloor N s /(2 r)\rfloor
\end{array}
$$

4. within the SI, a Candidate Set (CS) is compiled as follows:

- free slots are automatically included in the CS;

- the minimum size for the CS is $w_{C S \min }$. If less than $w_{C S \min }$ free slots are available in the SI, a suitable number of externally allocated ones must be included in it. These are selected starting from the ones allocated by the UEs more distant from the current transmitter;

- the designated $\mathrm{NTS}_{0}$, which we mark as $\sigma_{n t s_{0}}$, is randomly chosen from the ones in the CS with uniform probability, regardless of its state.

Finally, the UE transmits the NEP, to which it appends the offset between $\sigma_{n e p}$ and the $\sigma_{n t s_{0}}$. The UE subsequently waits for the $\sigma_{n t s_{0}}$, then moves to the next phase.

\subsubsection{The first frame phase}

The purpose of this phase is the reservation of the remaining $r-1$ NTSs beside NTS 0 , in order to ultimately allocate $r$ transmission slots per frame.

The procedure to reserve NTS $i+1$ must be performed before transmitting the packet in the NTS $i$, in order to be able to include in it the reservation information related to NTS $i+1$. This information includes, as illustrated in Fig. 4:

1. the offset (in slots) between NTS $i$ and NTS $i+1$;

2. the timeout $t_{i}$ related to NTS $i$.

The offset indicates the number of slots that separates NTS $i+1$ from NTS $i$. The timeout value represents the number of consecutive frames for which a NTS occupies one given slot. S-TDMA, in fact, is designed for highly mobile terminals: the slots chosen as NTSs thus need to be changed from time to time, in order to avoid merging collisions. Each terminal hence associates one timeout counter to every NTS, which is initialized with a random integer value between $t_{\min }$ and $t_{\text {max }}$ : ETSI standard values are $t_{\min }=3$ and $t_{\max }=7$. This means that after using a slot for a number of times equal to the timeout value initially attached to it, the reservation procedure must be repeated, and a new slot chosen to carry that NTS.

To reserve the next NTS, a procedure very similar to the one applied for selecting the NTS 0 is followed, until all of the $r$ NTSs have been selected. From a nomenclature standpoint, NSS for the SIs following the first one are referred to as Nominal Slots (NSs). Once this is done, the UE moves to the continuous operations phase.

\subsection{The continuous operations phase}

UEs stay indefinitely in the continuous operations phase after they reach it. In this phase, terminals perform their $r$ transmissions per frame in the previously allocated NTSs. At the same time, they continuously listen to the channel, in order to update their internal representation of the slots' states, which is a piece of information necessary to perform re-reservations. A 


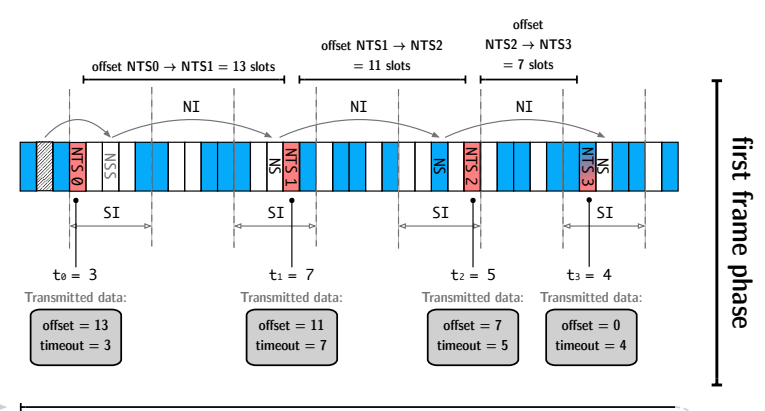

frame 1

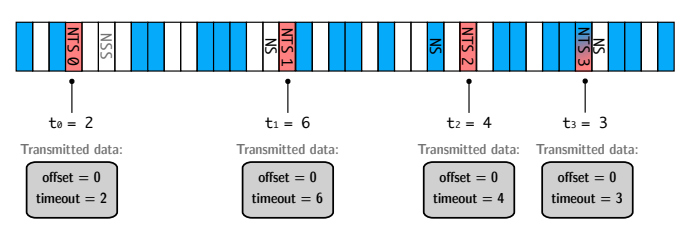

frame 2

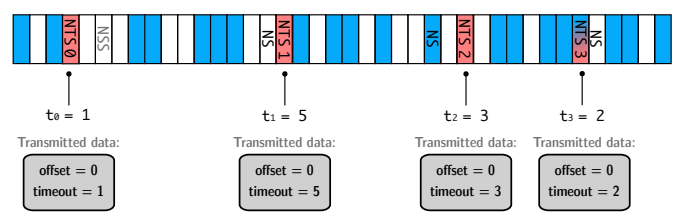

frame 3

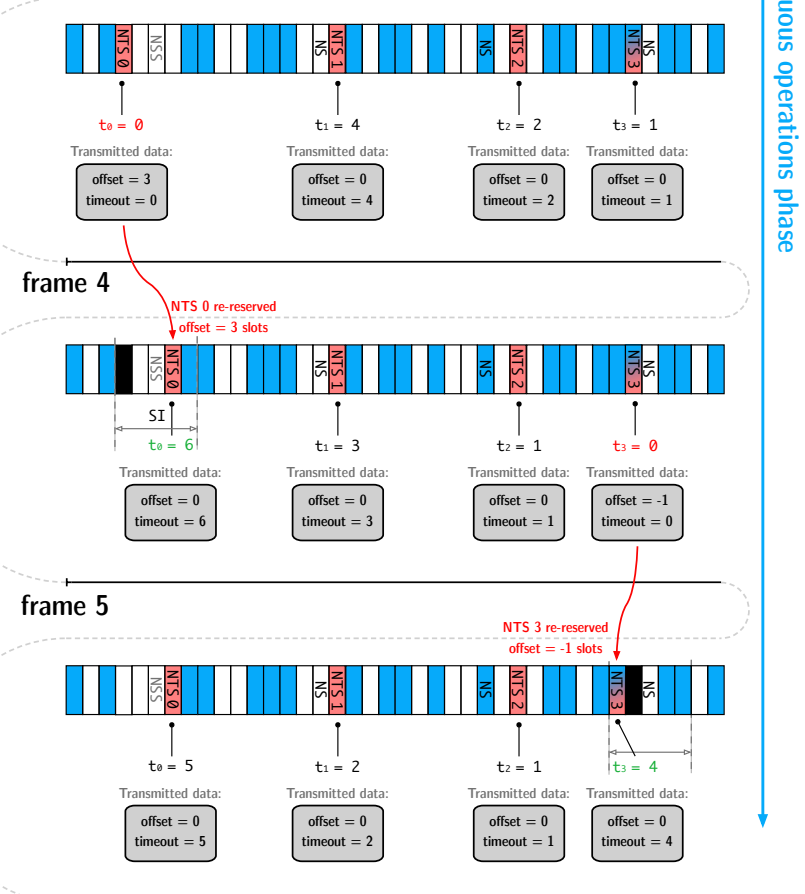

frame 6

time

Figure 4: S-TDMA, first frame phase and continuous operations phase.Free slots are colored in white, externally allocated slots are shaded in light blue, internally allocated slots are shaded in red. Slots shaded in both red and blue are reused slots. Unavailable slots are colored in black. re-reservation is the process with which a terminal selects and allocates a new slot for a NTS, after it has used it for the number of consecutive frames indicated by the timeout value. When the timeout for one NTS reaches 0 , a SI is chosen around the associated NS, a CS is constructed, and a novel NTS is chosen according to the same rules previously applied.

The continuous operations phase, along with the first frame phase, is illustrated in Fig. 4. It is worth noting that in the continuous operations phase, the semantics of the "offset" value is different from the first frame phase. It now indicates the offset between the position of one NTS in one frame and its position in the next one.

\section{Analytical model of S-TDMA}

\subsection{General model description}

The purpose of this work is to compute the asymptotic Slot Occupation Distribution (SOD) of S-TDMA as defined in [18], which is the probability distribution for one slot to be simultaneously utilized for transmission by $i$ users. Starting from the SOD, we can then derive the Packet Level Incoordination (PLI) [17], which is the probability for one transmission slot to be reused for transmission by more than one user. We evaluate the PLI as a function of the Offered Channel Load (OCL), a metric that represents the ratio between the number of slots per frame that would be required to satisfy the transmission requirement of all the UEs in the system and the number of slot actually available per frame. The OCL is mathematically defined in Section 4.4.

In order to achieve analytical tractability, we make the following abstractions:

- only the continuous operations phase is modeled: since we focus on evaluating the steady state behavior of the reservation process, we assume a network of UEs already aware of each other's presence;

- the CS is compiled without knowing the reservation pattern of concurrent users: instead, slots are added based on their probability of being sensed free;

- when necessary, externally allocated slots are included in the CS randomly, instead of being chosen based on the relative distance between the contending terminals;

- we assume a perfect Physical (PHY) layer, since our focus in on the MAC layer performance. It is worth noting that path loss and fading would indeed impair the probability of reception, but at the same time reduce the number of terminals competing for the same channel resources;

- terminals are either static or in homogeneous mobility.

To describe the behavior of S-TDMA we need to model two processes:

1. the timeout mechanism terminals associate to each NTS; 
2. the slot re-reservation mechanism.

Despite the protocol being deterministically structured, the distribution of the allocated slots and the choice of the NTSs are inherently random, making the state of the system at any point in time and space a random process. Both these processes will thus be described with stochastic models.

\subsection{Timeout process $\left(\pi_{t}\right)$}

The timeout mechanism determines for how many consecutive frames one user reserves each of its NTSs. When the counter reaches its minimum value, a re-reservation event is triggered and a new timeout is chosen. Assuming that users always have $r$ packets per frame to transmit, we can model the timeout process as a discrete-time Markov Chain, whose state diagram is represented in Figure 5. The time unit is 1 frame, since this is the counter update interval. Denoting with $\pi_{t, i}$ the

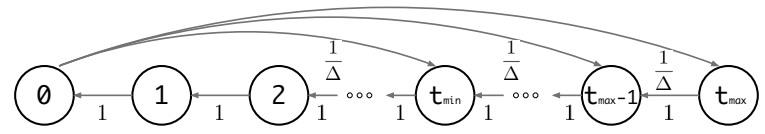

Figure 5: Markov Chain associated to the timeout mechanism

steady state probability for the counter to be in state $i$, we can compute the stationary distribution as follows:

$$
\begin{array}{lr}
\pi_{t, t_{\max }}=\pi_{t, 0} / \Delta & \\
\pi_{t, i}=\pi_{t, 0} / \Delta+\pi_{t, i+1} & t_{\text {min }} \leq i<t_{\text {max }} \\
\pi_{t, i}=\pi_{t, t_{\text {min }}} & 0 \leq i<t_{\text {min }}
\end{array}
$$

with $\Delta=t_{\max }-t_{\min }+1$. Imposing the normalization condition:

$$
\sum_{i=0}^{t_{\max }} \pi_{t, i}=1
$$

we can obtain $\pi_{t, 0}$, the probability for a slot re-reservation event to take place.

\subsection{Slot re-reservation process}

Let us consider an isolated network of $N_{t}$ UEs within each other's transmission range. Let us then consider a chunk $\chi$ of NI consecutive time slots: on average, we can assume that all of the $N_{t}$ users will need to transmit in exactly one of these NI slots. Among these NI, we identify one generic slot $\sigma_{t}$, we will refer to as the tagged slot. The re-reservation process, from the perspective of the tagged slot, can be modeled with the discretetime Markov chain depicted in Figure 6. Again, the time unit is 1 frame, being 1 frame the time interval between timeout counter value updates. The state variable $i$ represents the number of terminals currently reusing the tagged slot. The tagged slot will thus be free for $i=0$, allocated by a single user for $i=1$ and reused by multiple users when $i>1$. The extreme theoretical case is represented by the tagged slot being simultaneously allocated by all of the $N_{t}$ users, while all of the other NI -1 slots in $\chi$ are free. Denoting with $\pi_{i}$ the steady state

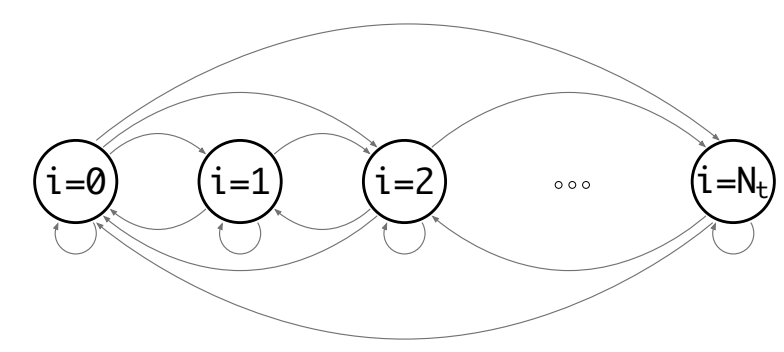

Figure 6: Markov Chain of the reservation process as seen by the tagged slot. The state variable $i$ is the number of terminals reusing the tagged slot.

probability for the tagged slot to be in state $i$, the stationary distribution $\pi=\left\{\pi_{0}, \ldots, \pi_{N_{t}}\right\}$ represents the SOD we are interested in.

Denoting with $p_{i, i+j}$ the probability for the process to transition from state $i$ to state $i+j$, the SOD can be computed solving the following equation:

$$
\pi=\pi \mathbf{P}
$$

where $\mathbf{P}$ is the transition probability matrix:

$$
\mathbf{P}=\left(\begin{array}{cccc}
p_{0,0} & p_{0,1} & \cdots & p_{0, N_{t}} \\
p_{1,0} & p_{1,1} & \cdots & p_{1, N_{t}} \\
\vdots & \vdots & \ddots & \vdots \\
p_{N_{t}, 0} & p_{N_{t}, 1} & \cdots & p_{N_{t}, N_{t}}
\end{array}\right)
$$

In order to compute a general expression for $p_{i, i+j}$ with $0 \leq$ $i \leq N_{t}, 0 \leq i+j \leq N_{t}$, we define the following events:

- an arrival in the tagged slot happens when a user in the process of re-reserving its transmission slot within $\chi$ choses the tagged slot as its NTS;

- a departure from a generic slot in $\chi$ happens when the timeout of one of the users that were previously allocating it expires, thus forcing that user to reserve a new NTS.

Since the timeout processes of the $N_{t}$ users are independent from each other, so are the departure events that involve them. Denoting with $D$ the number of departures that occur in $\chi$ and with $\bar{D}$ the number of departures that occur in the tagged slot, we have that the probability of having a total of $d$ departures in $\chi$ of which $\bar{d}$ are from the tagged slot while it is in state $i$ can be computed as:

$$
p_{i}\{\bar{D}=\bar{d} \mid D=d\}=\left(\begin{array}{c}
i \\
\bar{d}
\end{array}\right)\left(\begin{array}{c}
N_{t}-i \\
d-\bar{d}
\end{array}\right) \pi_{t, 0}{ }^{d}\left(1-\pi_{t, 0}\right)^{N_{t}-d}
$$

with $0 \leq \bar{d} \leq i$, as the number of departures from the tagged slot cannot exceed the number of users currently reserving it.

Let us now consider the arrival events in the tagged slot. For an arrival to occur, the following conditions must verify:

1. somewhere in $\chi$ one or more departures must occur;

2. one of the departing users' SI includes the tagged slot; 


$$
\begin{gathered}
p_{i}\{\bar{d}, a \mid d\}=p_{i}\{\bar{D}=\bar{d} \mid D=d\} \cdot p\{A=a \mid D=d\}= \\
\left(\begin{array}{l}
i \\
\bar{d}
\end{array}\right)\left(\begin{array}{c}
N_{t}-i \\
d-\bar{d}
\end{array}\right) \pi_{t, 1}{ }^{d}\left(1-\pi_{t, 1}\right)^{N_{t}-d} \cdot \sum_{k=a}^{d}\left(\begin{array}{l}
d \\
k
\end{array}\right) p_{S I}{ }^{k}\left(1-p_{S I}\right)^{d-k} \cdot\left(\begin{array}{l}
k \\
a
\end{array}\right) p^{a}(1-p)^{k-a}
\end{gathered}
$$

3. the tagged slot is included into the CS;

4. the tagged slot is chosen as the NTS.

The probability of having $A=a$ arrivals in the tagged slot must then be conditioned on having $d \geq a$ departures in $\chi$. The probability for the tagged user to belong to the SI of one generic re-reserving user is given by ${ }^{1}$ :

$$
p_{S I}=p\left\{\sigma_{t} \in S I\right\}=\min \left\{\frac{|S I|}{N I}, 1\right\}
$$

where $|S I|$ represents the cardinality of SI, i.e. the number of slots in SI. The probability to have $A=a$ arrivals in the tagged slot given that $d$ departures occur in $\chi$ can then be expressed as:

$$
\begin{aligned}
& p\{A=a \mid D=d\}= \\
& \sum_{k=a}^{d}\left(\begin{array}{l}
d \\
k
\end{array}\right) p_{S I}^{j}\left(1-p_{S I}\right)^{d-k}\left(\begin{array}{l}
k \\
a
\end{array}\right) p^{a}(1-p)^{k-a}
\end{aligned}
$$

where $p$ represents the probability for the tagged slot to be chosen by a re-reserving user as its next NTS, given that $\sigma_{t}$ belongs to that user's SI.

By combining (3) and (5) we can finally obtain the probability $p_{i}\{\bar{d}, a \mid d\}$ of having $a$ arrivals in the tagged slot and $\bar{d}$ departures from it, given that $d$ total departures happen in $\chi$ and $\sigma_{t}$ is in state $i$ as in equation (6).

Finally, the probability $p_{i, i+j}$ can be obtained as the sum of all the probabilities of the combinations of events such that the balance between the arrivals in the tagged slot and the departures from it is equal to $j$. Mathematically, this translates to:

$$
p_{i, i+j}=\sum_{C_{i, i+j}} p_{i}\{\bar{d}, a \mid d\}
$$

where the set of conditions $C_{i, i+j}$ is defined as follows:

$$
\begin{aligned}
& C_{i, i+j}: \\
& 0 \leq d \leq N_{t} \\
& 0 \leq \bar{d} \leq \min \{i, d\} \\
& 0 \leq a \leq d \\
& j=a-\bar{d}
\end{aligned}
$$

To be able to compute (7) we still need to determine $p$, that appears in (5) and (6). According to the S-TDMA protocol, the probability for a slot to be chosen as NTS by one re-reserving user (given that it belongs to its SI) depends on the state the tagged slot is sensed to be in.

\section{The tagged slot is sensed free}

If the tagged slot is sensed free, it is automatically included into the candidate set, from which the NTS is chosen with uniform probability. We then have that the probability for the tagged slot to be chosen while sensed free $p_{f}$ is:

$$
p_{f}=\frac{1}{\max \left\{\mathbb{E}\left[n_{f}\right], w_{C S \min }\right\}}
$$

where $\mathbb{E}\left[n_{f}\right]$ is the expected number of free slots within the SI and can be computed as:

$$
\mathbb{E}\left[n_{f}\right]=\sum_{k=1}^{|S I|} k \cdot\left(\begin{array}{c}
|S I| \\
k
\end{array}\right) \hat{p}^{k}(1-\hat{p})^{|S I|-k}
$$

with $\hat{p}$ being the probability for one slot to be sensed free.

\section{The tagged slot is sensed allocated}

If the tagged slot is sensed allocated, it only has a chance to be part of the candidate set of a re-reserving user if there are less than $w_{C S \min }$ free slots within its SI. Specifically, if $\overline{n_{f}}$ slots are free within the SI, with $\overline{n_{f}}<w_{C S \min }$, we have that:

$$
p\left\{\sigma_{t} \in S I \mid \text { allocated } \wedge \overline{n_{f}}\right\}=\frac{w_{C S \min }-\overline{n_{f}}}{|S I|-\overline{n_{f}}} .
$$

The probability for the tagged slot to be part of the CS while allocated is then:

$$
\begin{aligned}
& p\left\{\sigma_{t} \in S I \mid \text { allocated }\right\}= \\
& =\sum_{k=0}^{w_{C S \min }-1} \frac{w_{C S \text { min }}-k}{|S I|-k} \cdot\left(\begin{array}{c}
|S I| \\
k
\end{array}\right) \hat{p}^{k}(1-\hat{p})^{|S I|-k}
\end{aligned}
$$

Since the NTS is again chosen with uniform probability from the CS, the resulting probability $p_{a}$ for the tagged slot to be chosen while allocated is:

$$
p_{a}=\frac{1}{w_{C S \min }} \cdot \sum_{k=0}^{w_{C S \min }-1} \frac{w_{C S \min }-k}{|S I|-k} \cdot\left(\begin{array}{c}
|S I| \\
k
\end{array}\right) \hat{p}^{k}(1-\hat{p})^{|S I|-k}
$$

The probability $p$ in equations (5) and (6) must then be chosen between $p_{f}$ and $p_{a}$ according to the state the tagged slot is sensed being in. Since a slot is sensed free when, during the previous frame:

- it was free and no other user reserved it;

- it was allocated by $i$ users and all of them have to perform a re-reservation in the current frame;

\footnotetext{
${ }^{1} \min$ operation is necessary as it can be $|S I|>N I$ when $s=1$ in (1).
} 
in (5) and (6) we must set:

$$
\begin{cases}p=p_{f} & \text { if } i=0 \vee \bar{d}=i \\ p=p_{a} & \text { otherwise. }\end{cases}
$$

Following the same principle we can finally determine $\hat{p}$, the probability for a slot to be sensed free, as follows:

$$
\hat{p}=\sum_{k=0}^{N_{t}} \pi_{k}^{(f f)} \cdot \pi_{t, 0}{ }^{k}
$$

where $\pi_{k}^{(f f)}, 0 \leq k \leq N_{t}$ is the resulting SOD after that all the $N_{t}$ users have finished their first frame phase, entering the network. By computing (2), we can evaluate the asymptotic performance of the S-TDMA protocol configuration evolving from an initial state distribution $\boldsymbol{\pi}^{(f f)}=\left\{\pi_{0}^{(f f)}, \ldots, \pi_{N_{t}}^{(f f)}\right\}$.

Finally, we can compute the Packet Level Incoordination experienced by a user allocating the tagged slot as the probability for the tagged slot to be reused by more than 1 terminal:

$$
P L I=\sum_{k=2}^{N_{t}} \pi_{k}
$$

\subsection{Initial state}

The initial state distribution $\pi^{(f f)}=\left\{\pi_{0}^{(f f)}, \ldots, \pi_{N_{t}}^{(f f)}\right\}$ is computed based on the state of the system after all the terminals have finished the first frame phase. This depends on the OCL, which is the ratio between the number of slots required by the $N_{t}$ users, each transmitting $r$ packets per frame, and the number $N$ of available slots: $O C L=N_{t} \cdot r / N$.

The initial state is a system assumption: in this work we assume that if $N_{t}<=N$, no transmission slot is reused. When $N_{t}>N$, on the other hand, slots are chosen so to minimize the number of users reusing the same slot. $\boldsymbol{\pi}^{(f f)}$ after the first frame phase is thus computed as in equation (12).

$$
\left\{\begin{array}{l}
k=\left\lfloor N_{t} \cdot r / N\right\rfloor \\
\pi_{k+1}^{(f f)}=N_{t} \cdot r / N-\left\lfloor N_{t} \cdot r / N\right\rfloor \\
\pi_{k}^{(f f)}=1-\pi_{k+1}^{(f f)} \\
\pi_{j}^{(f f)}=0 \quad \forall j \neq k \vee j \neq k+1
\end{array}\right.
$$

\section{S-TDMA Analytical model: results}

In this section, we will show the algorithm outputs for the initialization described in section 4.4. For the timeout counter value, we consider two scenarios:

1. the standard [13] values $t_{\min }=3$ and $t_{\max }=7$;

2. the extended values $t_{\min }=1$ and $t_{\max }=10^{5}$.

Case 2. is meant to reduce the spurious collisions due to simultaneous allocation of the same slot by multiple users. This phenomenon, virtually inexistent in the S-TDMA protocol, affects our model due to the hypothesis of no knowledge of the concurrent reservation patterns. By comparing the values with those obtained in scenario 1 . we can evaluate its impact.

\subsection{Slot Occupation Distribution}

We compute the SOD for a system with $N=860$ slots/frame, a value very close to the 859 slots/frame established in [8] for 800 bytes CAMs, that allows us to obtain exactly $O C L=50 \%$ with 43 users having a report rate $r=10$ packets/s, and $O C L=$ $100 \%$ with the same 43 users transmitting $r=20$ packets/s. Both scenarios a) and b) have been obtained with $w_{C S \min }=1$.

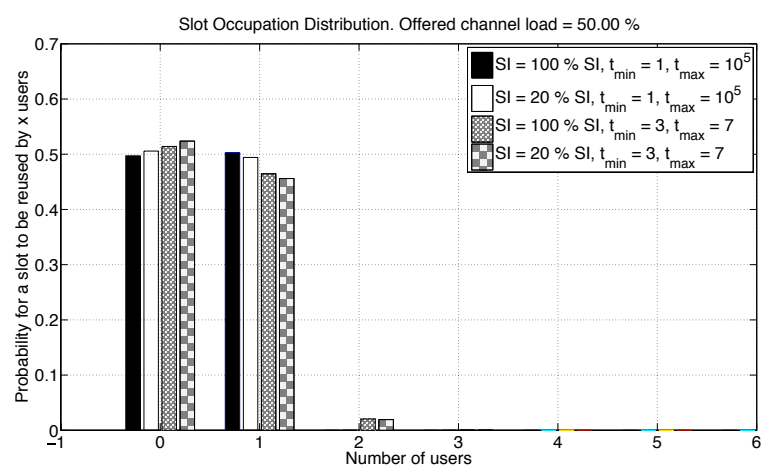

Figure 7: SOD for OCL $=50 \%: N=860, N_{t}=43, r=10$

In Fig. 7 can observe that for low OCL, the algorithm describes quite well the expected behavior of the protocol. The size of the SI has little influence, since enough free slots are available in the CS for both configuration. When the standard values $t_{\text {min }}=3$ and $t_{\text {max }}=7$ are used, a small probability of reuse by 2 users is observed, due to the effect of simultaneous reservations of the same slot by multiple users.

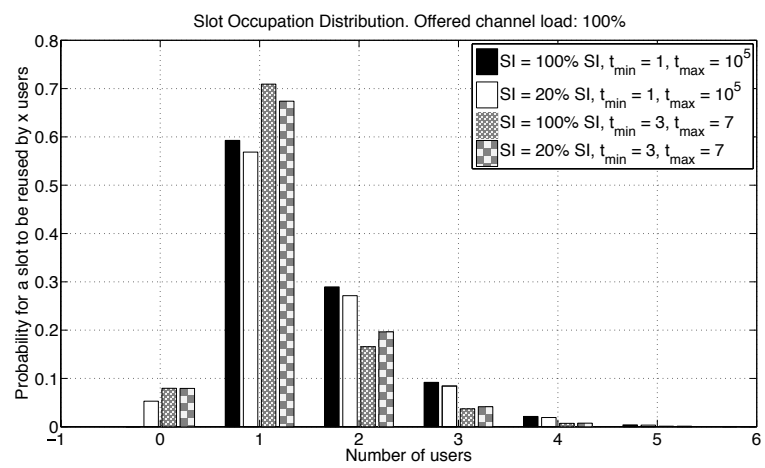

Figure 8: SOD for OCL $=100 \%: N=860, N_{t}=43, r=20$

In Fig. 8, the same scenario is evaluated for $O C L=100 \%$. In this case, we can observe that the output of the algorithm is less close to the ideal expected value of $\pi_{1}=1, \pi_{i}=0, i \neq 1$. This behavior can be attributed to the algorithm being based a random process trying to model a deterministic phenomenon. In this specific scenario, we can observe how the SOD for timeout values $t_{\min }=3$ and $t_{\max }=7$ provide the closest results to the expected behavior. This is due to the way the probability for a slot to be sensed free is computed in (10). A larger timeout counter range eliminates the occurrence of simultaneous reservation, with the side effect of drastically reducing the value of 
$\pi_{t, 1}$. As a consequence, the algorithm is less efficient in capturing the free slots left by departures.

It is worth noting that an endless reservation scenario can be obtained by setting $t_{\text {min }}=t_{\text {max }} \rightarrow \infty$. In this scenario, we obtain $\pi_{t, 1}=0$, which substituted in (7) and then in (2) provides a transition probability matrix $P$ equal to the identity matrix. In such a scenario, no re-reservation is observed, and slots that are in a given state at the end of the first frame phase keep remaining in the same state.

\subsection{Packet Level Incoordination}

In this section, we show the PLI in terms of its complementary, the Packet Level Coordination, or $(1-P L I)$, the probability for one user not to have one transmission slot reused by other terminals. This metric is evaluated for scenario a) in Fig. 9 and for scenario b) in Fig. 10. In both cases, we consider $N_{t}=43$ users, with report rates swiping from $r=1$ to $r=40$ packets/s to obtain increasing values of OCL.

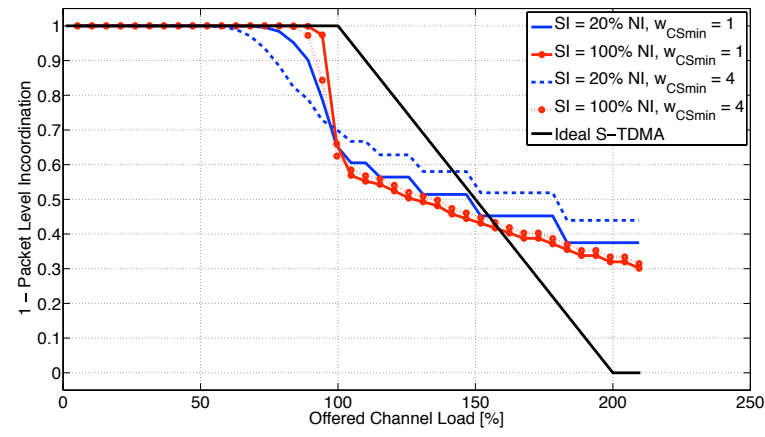

Figure 9: Packet Level Coordination for $t_{\min }=1$ and $t_{\max }=10^{5}$

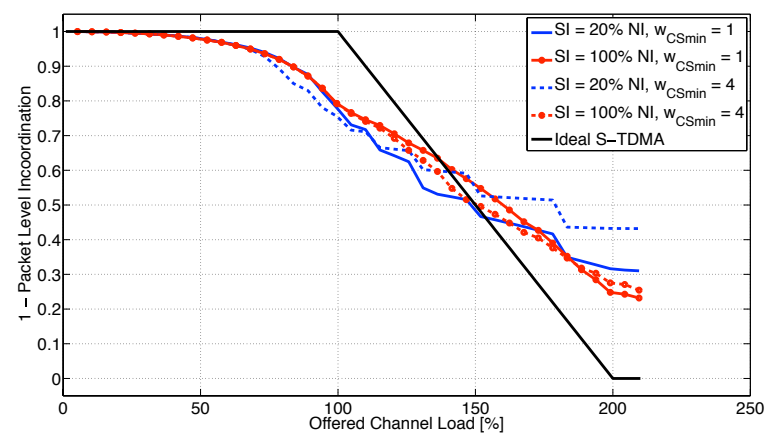

Figure 10: Packet Level Coordination for $t_{\min }=3$ and $t_{\max }=7$

We can observe that the configuration with extended timeout counter range offers results closer to the ideal curve for $O C L \leq 100 \%$, but then it diverges from it for higher OCL values. On the other hand, the configuration with the standard values diverges from the expected curve earlier, but then, for higher OCL, the difference is lower. This behavior can again be attributed to the computation of the probability for a slot to be sensed free, which cannot efficiently capture the slots left free by re-reserving users in highly loaded scenarios.
The curves obtained for wider values of the minimum candidate set size $w_{C S \min }$ introduce further impairment for higher OCL: this is originated by the allocated slots that have to be included in the CS, which can be chosen with equal probability than the already scarce free slots.

\section{S-TDMA for Cellular-V2X}

S-TDMA has originally been designed for pure time slot resource allocations (Fig. 2). In this section we describe how to apply S-TDMA to the C-V2X frequency-time slots configuration, illustrate the impairment effect due to half duplex in $\mathrm{C}-\mathrm{V} 2 \mathrm{X}$, and propose extensions to mitigate it.

\subsection{S-TDMA on $C$-V2X}

As opposed to $802.11 \mathrm{p}$, wherein slots are multiplexed in time domain only, C-V2X PHY layer enables the disposition of multiple transmission slots within the same bandwidth. Different slots configurations are possible: the same number of Resource Blocks (RBs) can be assigned predominantly in time domain (Fig. 11a), or predominantly in frequency domain (Fig. 11b). The advantage of the former is a better slot mapping at

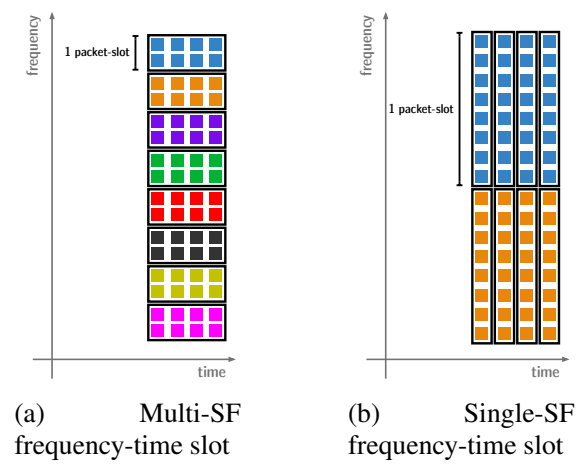

Figure 11: S-TDMA Slot mappings for C-V2X

the cost of a longer transmit time, while the latter provides a shorter transmit time at the cost of spectrum agility, which is preferred for delay-critical safety-related packets. Moreover, this latter configuration helps minimizing the effects of the HD impairment. The time-frequency arrangement of slots in C$\mathrm{V} 2 \mathrm{X}$, in fact, makes S-TDMA vulnerable to the impairment due to HD, which is still a technological limitation for nowadays transceivers. This means that a UE will not be able to receive the packets transmitted by other UEs in slots temporally co-located with its NTSs, as illustrated in Fig. 12. This further source of packet loss could be detrimental for S-TDMA, since packets carry the information necessary for the UEs to reserve their transmission slots: these loss could hence lead to recurring collisions. In this paper, we thus present two protocol extensions, S-TDMA for OFDMA deployments (OSTDMA) and Selective Hiding S-TDMA (SH-STDMA), whose aim is to mitigate the effects of HD impairment. 


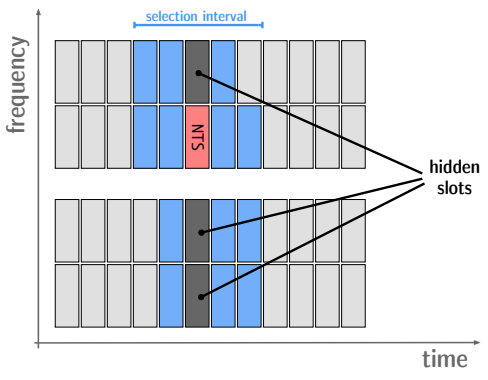

Figure 12: hidden slots due to half duplex transceivers

\subsection{S-TDMA Extensions}

We first need to introduce a new "hidden' state for the slots, which is associated to those slots that cannot be received by a given UE because one of its NTSs is located within the same subframe (as shown in Fig. 12). OSTDMA and SH-STDMA both retain the same protocol structure of S-TDMA, but alter the way the candidate set is compiled.

\subsubsection{OSTDMA}

OSTDMA is identical to S-TDMA, except for the fact that hidden slots are excluded from the candidate set. This is done to avoid scheduling a new NTS on a slot which might be reserved, but not detectable.

\subsection{2. $S H$-STDMA}

SH-STDMA takes a different approach to dealing with the half duplex impairment. Since HD reception losses are due to a technological limitation of the transceiver, they are unavoidable: the protocol can however intelligently reduce its negative effects.

SH-STDMA is conceived to selectively avoid hiding the NTS allocated by vehicles closer to the transmitter, as the information transmitted by these is the most relevant. To do this, the procedure of compilation of the candidate set is modified as follows:

1. the selection interval is compiled as in S-TDMA

2. A penalty is assigned to all the LTE subframes completely or partially within the SI. By denoting with $S_{i}$ the $i^{\text {th }}$ in the SI, with $\sigma_{j}$ the $j^{\text {th }}$ slot within a given subframe, and with $n_{s}$ the number of slots within a subframe, the penalty $P_{i}$ for the $i^{t h}$ subframe is computed as in

$$
P_{i}=\sum_{j=0}^{n_{s}-1} g\left(d\left(\sigma_{j}\right)\right)
$$

where $g(x)$ is an arbitrary function decreasing with $x$, and $d\left(\sigma_{j}\right)$ is the distance between the current UE and the one which reserved slot $j$. In case the slot $j$ is free, $g(x)=0$.

3. a candidate set is compiled as follows:

- it must contain exactly $w_{C S m i n}$ slots (the minimum for S-TDMA)

- free slots are added starting from the subframes with lower penalty $P_{i}$
- if fewer than $w_{C S \min }$ free slots are available in the SI, the remaining slots are picked by progressively choosing them from the subframes with lower penalty, starting from those allocated by the farthest UEs. The unlikely case wherein fewer than $w_{C S \min }$ slots are viable to be part of the CS (i.e. they are not unavailable) is neglected in this work.

In the evaluation on the next section, we adopted the following penalty function:

$$
g\left(d\left(\sigma_{j}\right)\right)= \begin{cases}1 / d\left(\sigma_{j}\right) & \text { if } \sigma_{j} \text { is externally allocated } \\ 0 & \text { else }\end{cases}
$$

Fig. 13 illustrates the principle of SH-STDMA. On the left side, the SI is illustrated: in it, externally allocated slots are color-coded according to the distance between the UE reserving it and the current UE. Higher penalties are assigned to subframes containing slots reserved by closer vehicles.

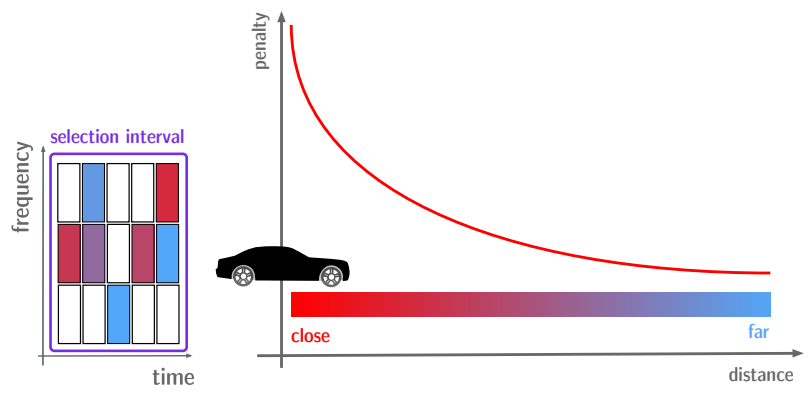

Figure 13: SH-STDMA, basic working principle. The slots on the left are colored based on the distance between from UE that reserved it. The curve represent an example of penalty function.

\section{Evaluation methodology and results}

We study the impairment due to half duplex on the S-TDMA reservation protocol by means of simulation. To this end, we implemented S-TDMA ${ }^{2}$ running on a custom developed discreteevents simulator, which allows us the flexibility to either abstract the PHY layer to only test the MAC layer phenomena, or to consider a realistic propagation scenario. This is important, since standardization on C-V2X is quickly evolving, and at any given time it is difficult to identify a convenient PHY configuration. It is worth noting that the simulated version

\subsection{Effect of HD impairment on S-TDMA}

We are first interested in comparing the fundamental behavior of S-TDMA, OSTDMA, and SH-STDMA in terms of PRR versus the OCL, a new metric we introduce. In any point in space, OCL represents the ratio between the slots that are necessary to satisfy the communication needs of all the UEs within

${ }^{2}$ it is worth noting this implementation of the protocol is not affected by the assumption made in Section 4.1, which were functional to developing the analytic model. 
radio range, and the total number of available slots. For instance, if there are 100 slots in a frame, and 5 UEs are in the network, each transmitting 10 packets per second, they would need 50 slots per frame to satisfy their communication needs: this results in a OCL of 0.5. OCL can indeed be larger than one, when more slots are needed than there are available.

In order to compare the fundamental behavior of the each protocols to increasing OCL, we consider a scenario wherein all of the UEs are within respective TX/RX range, in a ideal C-V2X channel configuration. Degradation due to propagation is thus not applied: the system's performance are hence limited only by collisions and by HD impairment. Increasing level of OCL are achieved by increasing densities of UEs: during the simulation, UEs are progressively dropped new in a random position within range of all of the previous ones. UEs then remain static in that position. The average time between insertions is based on the number of UEs in the system, to equalize the number of samples collected, and it varies between 4 and 80 seconds. The reaction of the protocols is then logged.

In Fig. 14, we first consider the curves for S-TDMA with and without (ideal S-TDMA) the HD impairment. All the curves in the figure have been obtained by averaging 120 simulation runs. Three OCL regions can be identified, wherein the proto-

Table 1: Protocol and channel configuration parameters

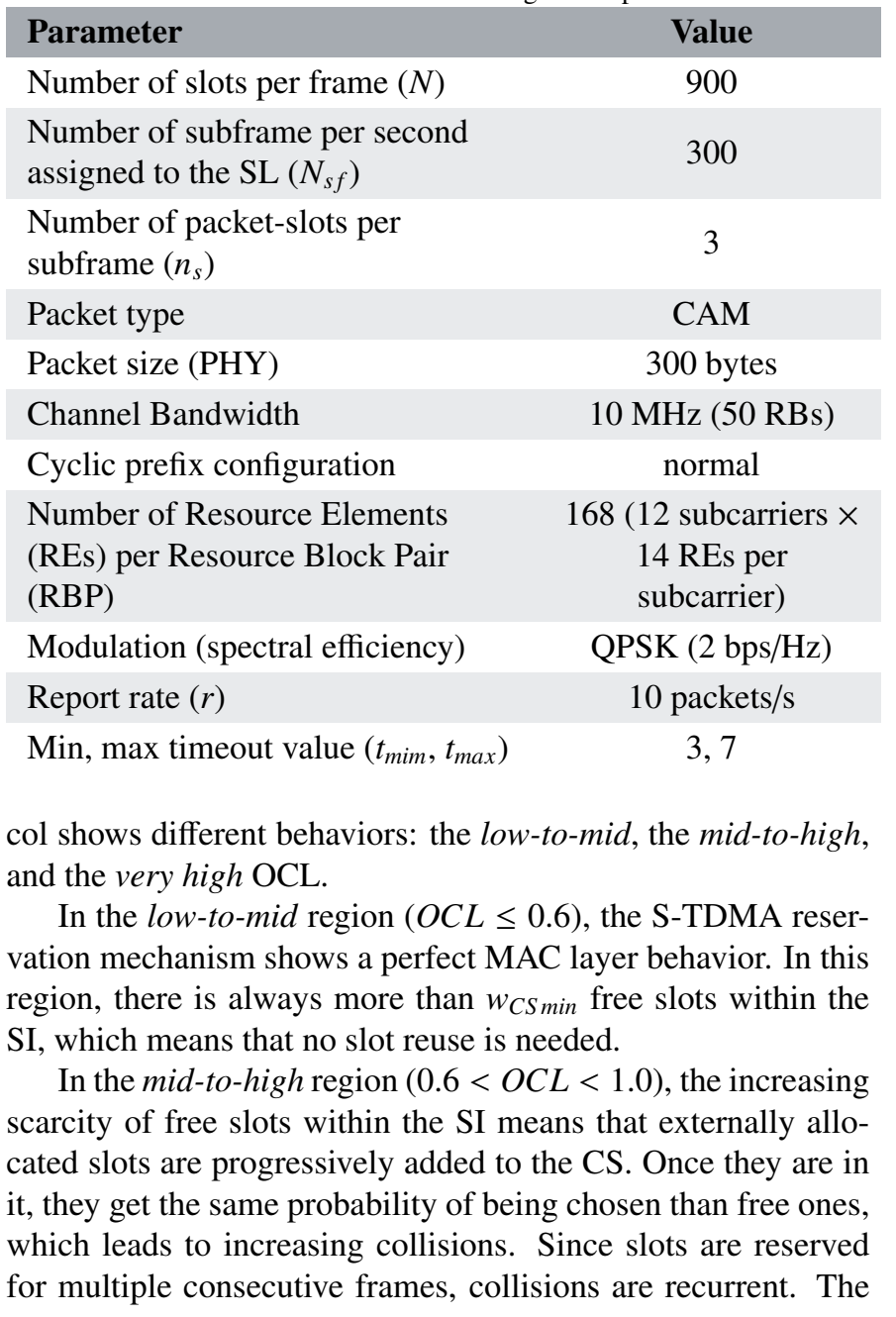

random nature of the selection is what causes the $95 \%$ confidence interval to be wider in this region, as raw performance is very much dependent on every instance.

In the very high region, the system is dominated by collisions, as free slots become very rare. In this extreme region, the behavior of the system becomes very predictable, as demonstrated by the very narrow $95 \%$ confidence region.

The effect of HD impairment on S-TDMA can be observed by comparison against the "ideal S-TDMA" wherein this effect is neglected. Starting from the very beginning of the low-to-mid region, the performance moves away from the ideal benchmark, as the reservation information losses also happen at lower OCL. To this, we must add losses that each UE suffers of the packets transmitted in slots that are within the same subframe (time colocated) to its NTSs. The mid-to-high region shows the largest gap, as the effects of progressive addition of externally allocated slots to the CS and the HD impairment are combined. In the very high OCL region, it is shown how the HD impairment becomes less and less relevant, as the system is dominated by collisions, by the fact that the curve superimposes with the ideal one.

\subsection{Evaluation of S-TDMA protocol extensions}

In Fig. 14, the performance of S-TDMA, OSTDMA, and SH-STDMA are compared in a LTE V2X channel system as in Table 1, against the ideal case. We observe that for lower OCL

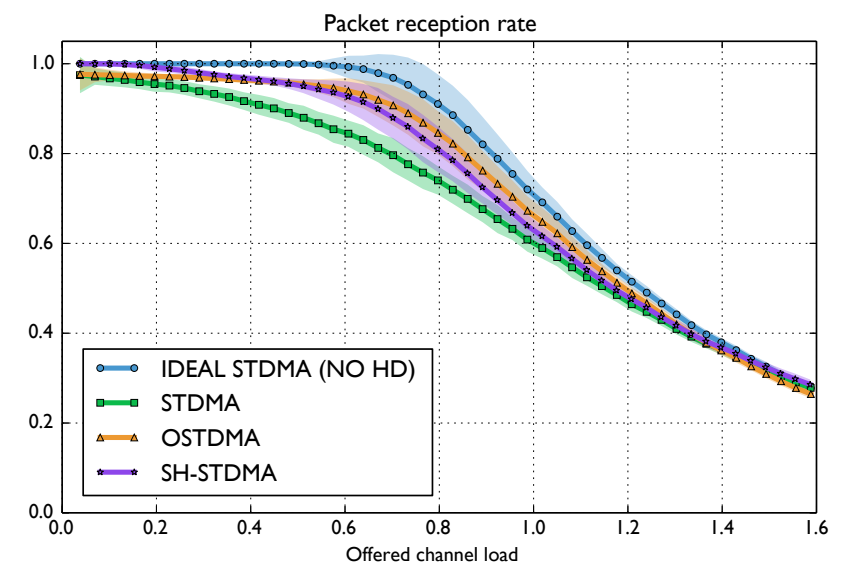

Figure 14: Protocol extensions: OSTDMA and SH-STDMA vs S-TDMA. The shaded area around the curves represents the $95 \%$ confidence interval

SH-STDMA provides optimal or near-optimal performance, thanks to its mechanism that avoids hiding users, allowing for a more efficient use of the channel's time dimension. When the OCL exceeds $25 \%$, it becomes increasingly difficult to have subframes within the CS that are entirely made of free slots. It is worth noting that the $25 \%$ figure is dependent of the ratio between $n_{s}$ and $N$. The considered scenario has the purpose of illustrating this phenomenon: realistic deployments may have larger $N$ (for instance, 3000 slots per second), and consequently larger SIs, which move this point at higher OCL.

On the other hand, at lower OCLs, OSTDMA provides performance not too dissimilar from S-TDMA, since in this region 
performance are essentially affected by missed reception of hidden slots. The missed reception of reservation information is less relevant, due to the high availability of free slots in the CS, hence the lower probability of contemporary reservation of the same slot by multiple UEs.

In the mid-to-high region, one can observe a crossing between the SH-STDMA and the OSTDMA curves, with the latter starting to performing slightly better than the former. This phenomenon is very dependent on this specific simulation scenario, and might be attributed to the choice of function $g(x)$ made for SH-STDMA, which does not assign a penalty to unavailable slots, those affected by collisions. The purpose of this perfect PHY configuration was to isolate the MAC layer performance; in a realistic implementation, however, those are also affected by the behavior of lower layers. Specifically, when fading is considered, in case of collision, the transmission coming from the closest UEs is likely to be received thanks to capture effect. In the scenario considered in this section, on the other hand, any kind of slot reuse makes a slot unavailable, hence considered by SH-STDMA as a viable slot to be hidden, by scheduling the NTS in a free slot located within the same subframe.

In the very high OCL region, the system is dominated by collisions, making all the scheduling systems perform equally, and equal to the ideal case.

\subsection{Analysis of packet loss causes}

We are now interested in is breaking down the causes of the packet losses, versus the distance between TX and RX. Since many factors are involved, we performed this analysis by simulating a realistic scenario with mobile users and a channel propagation model, as described in Table 2. We considered a highway scenario, with a relatively high vehicle density (240 per $\mathrm{km}$ ), with highly mobile users. As in [7] we apply Nakagami$\mathrm{m}$ fading over a two slopes propagation model. In this scenario we identify four possible causes for packet loss: (i) "collision", which occurs when multiple packets are transmitted on the same slot, and are received with SINR so low to not allow the decoding of either; (ii) "Path loss", wherein no collision occurs, but the SINR is degraded below decoding capability due to propagation; (iii) "Another stronger transmission" occurs when multiple transmission happen on the same slot, and one of them has SINR high enough for it to be decoded, but it is not the one considered; (iv) "hidden", when none of the previous occur, and a otherwise successful reception is made impossible by HD. The relative effect on total global packet losses are depicted for S-TDMA and SH-STDMA in Fig. 15a, and Fig. 15b respectively. The curves have been obtained by averaging 10 simulation run, each containing data logged for 120 seconds, after 50 seconds of transient during which UEs are started up at random times, to cancel out spurious transient effects. By comparing them, we can observe how SH-STDMA successfully mitigates the effect of HD impairment for close-by vehicles, reducing the incidence of hidden slots on packet losses by $40 \%$ w.r.t. S-TDMA. The effects of propagation appear to be independent on the protocol; on the other hand the increase of

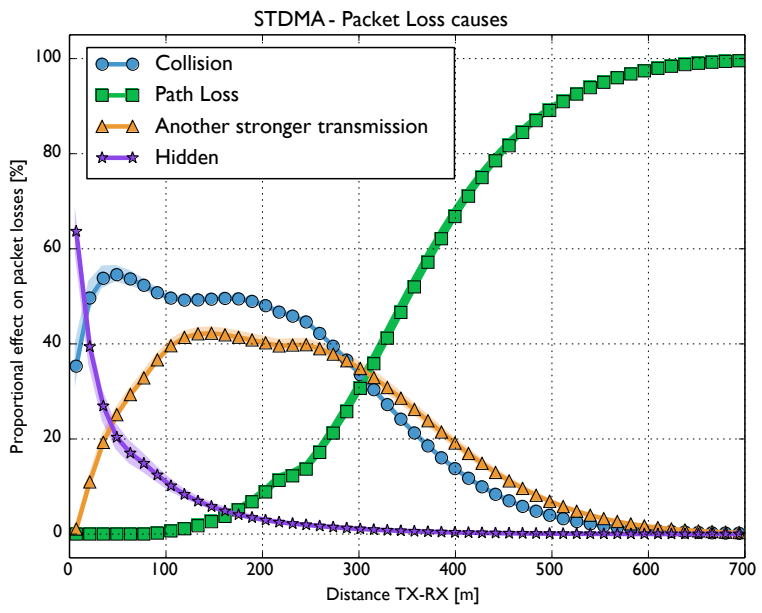

(a) S-TDMA

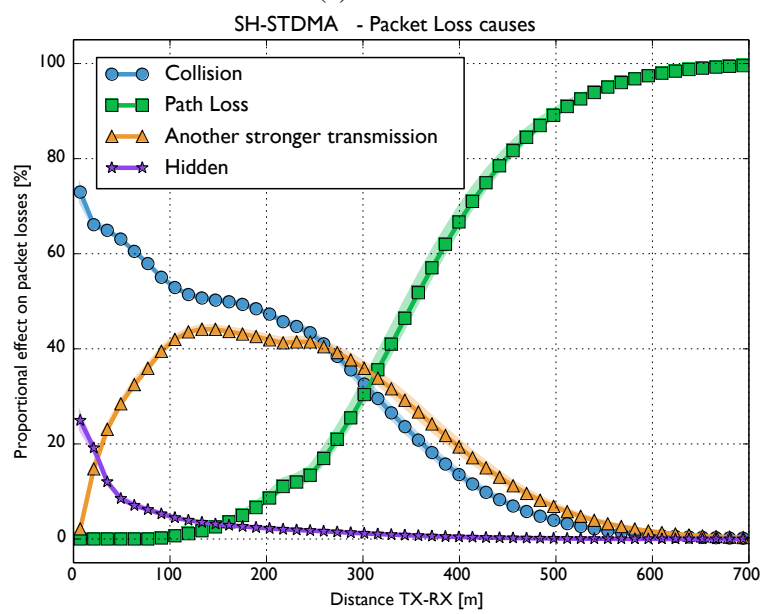

(b) SH-STDMA

Figure 15: Causes of packet loss vs TX-RX distance

collisions for shorter distances in SH-STDMA is due to the nature of the protocol itself: for higher densities, when less fewer slots are available, S-TDMA starts adding externally allocated ones to the CS, which can be chosen with equal probability to the free ones. Since SH-STDMA adds further restrictions on the slots that can be in the CS, this phenomenon is more likely to occur in high density deployments.

\section{Conclusion and future works}

Ad-hoc connectivity (mode 4) on the sidelink will represent a fundamental component of C-V2X, since it will enable crossoperator low-latency broadcast of safety-critical messages. It is thus important to adopt a distributed scheduling algorithm that provides high packet reception rate and stable performance under any channel load condition. In this paper we advocate for S-TDMA, an algorithm already extensively studied for $802.11 \mathrm{p}$ and commercially deployed. In this paper, we showed analytically that S-TDMA achieves high and stable packet reception performance by design. We then evaluated the impact due to half duplex transceivers, and propose two protocol extensions for S-TDMA to reduce its impairment. The remarkable perfor- 
Table 2: Mobility and propagation parameters

\begin{tabular}{lc} 
Mobility parameter & Value \\
Configuration & Highway, 6 lanes/direction \\
\hline Length & $2 \mathrm{~km}$ (with wrap-around) \\
Average speed per lane $[\mathrm{m} / \mathrm{s}]$ & $23,23,30,30,37,37$ \\
\hline $\begin{array}{l}\text { Density } \\
\text { Propagation/protocol } \\
\text { parameter }\end{array}$ & 240 [vehicles/km] \\
Central frequency & Value \\
Two slopes path loss model & $\gamma_{1}=2.1$ for $d<100 \mathrm{~m}$, \\
\hline $\begin{array}{l}\text { Fading } \\
\text { Transmission power }\end{array}$ & 3.8 for $d>100 \mathrm{~m}$ \\
\hline $\begin{array}{l}\text { Number of slots per frame } \\
(N)\end{array}$ & Nakagami-m \\
\hline
\end{tabular}

mance that can be achieved on the sidelink by them, make STDMA-based protocol valid candidates to support V2X safety critical transmissions over the sidelink.

In future, we envision to perform the evaluation in an even more realistic configuration, by implementing the protocols in OpenAirInterface [21], which recently was extended with the support of a standard-compliant implementation of the sidelink. Furthermore, the proposed protocols will be compared with the 3GPP Semi Persistent Scheduling will be performed, once a final standardized version of it will be available.

\section{Acknowledgments}

This work has been funded in part by the EU H2020 HIGHTS project (grant no. 636537), and in part by the French-Germany Academy for the Industry of the Future with the SCHEIF Project. EURECOM acknowledges the support of its industrial members, namely, BMW Group, IABG, Monaco Telecom, Orange, SAP, and Symantec.

\section{References}

[1] 5G-PPP, 5G Automotive Vision white paper, Technical Report, 2015.

[2] M. Hadded, P. Muhlethaler, A. Laouiti, R. Zagrouba, L. A. Saidane, Tdma-based mac protocols for vehicular ad hoc networks: A survey, qualitative analysis, and open research issues, IEEE Communications Surveys Tutorials 17 (2015) 2461-2492.
[3] F. Yu, S. Biswas, A self reorganizing mac protocol for inter-vehicle data transfer applications in vehicular ad hoc networks, in: 10th International Conference on Information Technology (ICIT 2007), pp. 110-115.

[4] H. A. Omar, W. Zhuang, L. Li, Vemac: A tdma-based mac protocol for reliable broadcast in vanets, IEEE Transactions on Mobile Computing 12 (2013) 1724-1736.

[5] R. Zou, Z. Liu, L. Zhang, M. Kamil, A near collision free reservation based mac protocol for vanets, in: 2014 IEEE Wireless Communications and Networking Conference (WCNC), pp. 1538-1543.

[6] R. Scopigno, H. A. Cozzetti, Mobile slotted aloha for vanets, in: 2009 IEEE 70th Vehicular Technology Conference Fall, pp. 1-5.

[7] K. Sjöberg, Medium Access Control for Vehicular Ad Hoc Networks, Ph.D. thesis, Chalmers University of Technology, 2013.

[8] ETSI, TR 102861 Intelligent Transport Systems (ITS); STDMA Recommended Parameters and Settings for Cooperative ITS; Access Layer Part, Technical Report, ETSI, 2012.

[9] ETSI, TR 102862 Intelligent Transport Systems (ITS); Performance Evaluation of Self-Organizing TDMA as Medium Access Control Applied to ITS; Access Layer Part, Technical Report, ETSI, 2011.

[10] Istituto Superiore Mario Boella, MS-Aloha: A wireless protocol specific for vehicular communications and suitable for automation (http://www.ms-aloha.eu/), 2011.

[11] H. A. Cozzetti, R. M. Scopigno, L. Casone, G. Barba, Comparative analysis of ieee 802.11p and ms-aloha in vanet scenarios, in: 2009 IEEE Asia-Pacific Services Computing Conference (APSCC), pp. 64-69.

[12] X. Wu, J. Li, R. M. Scopigno, H. A. Cozzetti, Insights into possible vanet 2.0 directions, in: Vehicular ad hoc Networks, Springer, 2015, pp. 411-455.

[13] ITU, Rec. M.1371-5 - Technical characteristics for an automatic identification system using time-division multiple access in the VHF maritime mobile band, International Telecommunications Union, 2014.

[14] A. Alonso, C. Mecklenbrauker, Stabilization Time Comparison of CSMA and Self-Organizing TDMA for Different Channel Loads in VANETs, in: ITS Telecommunications (ITST), 2012 12th International Conference on, pp. 300-305.

[15] K. Sjoberg, E. Uhlemann, E. G. Strom, How severe is the hidden terminal problem in vanets when using csma and stdma?, in: 2011 IEEE Vehicular Technology Conference (VTC Fall), pp. 1-5.

[16] K. S. Bilstrup, E. Uhlemann, E. G. Strom, Scalability issues of the mac methods stdma and csma of ieee 802.11p when used in vanets, in: 2010 IEEE International Conference on Communications Workshops, pp. 1-5.

[17] T. Gaugel, J. Mittag, H. Hartenstein, S. Papanastasiou, E. G. Strom, Indepth Analysis and Evaluation of Self-Organizing TDMA, in: Vehicular Networking Conference (VNC), IEEE, pp. 79-86.

[18] T. Gaugel, J. Mittag, H. Hartenstein, E. G. Strom, Understanding differences in MAC performance, A World of Wireless, Mobile and Multimedia Networks (WoWMoM), 2014 IEEE 15th International Symposium on (2014) 1-6.

[19] ETSI, TS 102 637-2 Intelligent Transport Systems (ITS); Vehicular Communications; Basic Set of Applications; Part 2: Specification of Cooperative Awareness Basic Service, Technical Report, ETSI, 2011.

[20] L. Gallo, J. Härri, Analytical study of self organizing TDMA for V2X communications, in: DVC 2015, 1st IEEE ICC Workshop on Dependable Vehicular Communications, London, UK.

[21] N. Nikaein, M. K. Marina, S. Manickam, A. Dawson, R. Knopp, C. Bonnet, Openairinterface: A flexible platform for $5 \mathrm{~g}$ research, SIGCOMM Comput. Commun. Rev. 44 (2014) 33-38. 